\title{
Schreckgespenst Impact-Faktor
}

Impact-Faktor. Es gibt wohl kaum ein Wort, das den Medizinwissenschaftler so elektrisiert wie dieses, das die Gemüter erregt und das ähnlich kontrovers diskutiert wird. Wer sich habilitieren möchte, wer sich gar um einen Lehrstuhl bewirbt, der weiss, dass er an einer Zahl mit Sicherheit nicht vorbeikommen wird: dem Impact-Faktor, zu deutsch etwa Bedeutungsfaktor (impact $=$ bleibender Eindruck, der bei einem Aufprall entsteht).

Worum geht es? Eine amerikanische Firma mit Namen ISI (Institute for Scientific Information) gibt seit vielen Jahren den sog. SCI (Science Citation Index) heraus, eine Datenbank, die in etwa der bekannten Datenbank Medline vergleichbar, aber thematisch noch breiter angelegt und unter dem Namen «Current Contents» wohl vielen geläufig ist. Das ISI berechnet nun für jede im SCI erfasste Zeitschrift den Impact-Faktor, indem die Literaturverzeichnisse aller Publikationen aller übrigen erfassten Zeitschriften danach durchforstet werden, ob bzw. wie häufig eine Publikation aus den vorangegangenen zwei Jahren aus einer bestimmten Zeitschrift zitiert wurde. Diese Anzahl wird dann in Bezug gesetzt zur Gesamtzahl der in dieser Zeitschrift in den vorangegangenen zwei Jahren erschienenen Publikationen und fertig ist der ImpactFaktor [1]. Mathematisch formuliert hiesse die Formel für unsere Zeitschrift also: Impact-Faktor 2001 = im Jahr 2001 in allen übrigen im SCI gelisteten Zeitschriften zitierte Publikationen der FORSCHENDEN KOMPLEMENTÄRMEDIZIN UND KLASSISCHEN NATURHEILKUNDE der Jahre 1999 und 2000 geteilt durch die Gesamtzahl der zitierfähigen Publikationen in der FORSCHENDEN KOMPLEMENTÄRMEDIZIN UND KLASSISCHEN NATURHEILKUNDE der Jahre 1999 und 2000. Ein Impact-Faktor von 1 bedeutet demnach, dass rechnerisch jede veröffentlichte Arbeit innerhalb von 2 Jahren einmal in einer anderen im SCI gelisteten Zeitschrift zitiert wurde und ein Impact-Faktor von 0,2 , dass rechnerisch jede fünfte veröffentlichte Arbeit innerhalb von 2 Jahren einmal in einer anderen im SCI gelisteten Zeitschrift zitiert wurde.
Hinter dieser einfachen Arithmetik steckt die Idee, dass gute und relevante Arbeiten häufig und wenig bedeutsame bis schlechte Arbeiten selten zitiert werden. Zeitschriften, aus denen häufig Arbeiten zitiert werden, haben, der Logik folgend, wohl einen vergleichsweise hohen Anteil an guten Arbeiten. Das wiederum macht sie als Publikationsforum interessant, was sich in einer besonders hohen Anzahl eingereichter Arbeiten manifestiert, aus denen dann wiederum die besten ausgewählt werden können. Und das wiederum macht sie für den Leser attraktiv, weshalb Publikationen in solchen Zeitschriften eben mehr «Impact» haben.

Ein Impact-Faktor von 1 ist etwa die Schwelle, ab der eine Zeitschrift als «international bedeutend» angesehen wird. Spitzenreiter unter den klinischen Zeitschriften sind das «New England Journal», der «Lancet», «JAMA», und die «Annals of Internal Medicine» mit einem Impact-Faktor von etwa 10 bis 25; Zeitschriften, in denen Ergebnisse aus der Grundlagenforschung publiziert werden, kommen teilweise auf noch wesentlich höhere Impact-Faktoren (ohne dass sie allgemein sehr bekannt wären). Unter den deutschsprachigen klinischen Zeitschriften gibt es nur wenige, die an einen Impact-Faktor von 1 heranreichen oder ihn gar überschreiten, und die FORSCHENDE KOMPLEMENTÄRMEDIZIN UND KLASSISCHE NATURHEILKUNDE sitzt mit einem aktuellen Impact-Faktor von 0,6 eindrucksvoll «in der ersten Reihe».

Warum aber wird dieser Impact-Faktor so kontrovers diskutiert? Da gibt es einige Probleme, die grundsätzlicher Natur sind [2]; das wichtigste für uns in Deutschland ist sicherlich die Sprache [3]. In Englisch publizierte Arbeiten können weltweit gelesen (und, falls für gut befunden, zitiert) werden, deutschsprachige Arbeiten etwa sind im Wesentlichen auf den deutschen Raum beschränkt; sie haben deshalb natürlich weltweit auch nur einen begrenzten Impact (was das ISI misst), können aber innerhalb Deutschlands gleichwohl viel bewegen (was sich im SCI so gut wie nicht abbildet).

Ein weiteres Problem liegt darin, dass selbst die «besten» Zeitschriften in kleinen Fachgebieten, wie z.B. der Augenheil-

\section{KARGER}

Fax +497614520714

E-mail Information@Karger.de www.karger.com/journals/fkm

\section{(c) 2002 S. Karger GmbH, Freiburg}

Accessible online at:

www.karger.com 
kunde oder der Sportorthopädie, nur einen bescheidenen Impact-Faktor haben. Dies ganz einfach deshalb, weil nur eine begrenzte Anzahl von Forschungsgruppen existiert, die nur eine begrenzte Anzahl von Publikationen produzieren kann und nur darin werden selbst bahnbrechende Arbeiten auf dem jeweiligen Gebiet zitiert.

Der Teufel steckt also im Detail, und es fehlt nicht an Vorschlägen für Korrekturfaktoren usw. [4]. Gegen ein immer wieder vorgebrachtes Argument, der Impact-Faktor sei «grundsätzlich untauglich» und sollte deshalb am besten ignoriert werden, steht wohl die Macht des Faktischen.

Für den Bereich der Naturheilkunde liegt im Impact-Faktor durchaus auch eine Chance. Die Tatsache, dass der ImpactFaktor der FORSCHENDEN KOMPLEMENTÄRMEDIZIN UND KLASSISCHEN NATURHEILKUNDE in wenigen Jahren so stark gestiegen ist und ein inzwischen wirklich beachtliches Niveau erreicht hat, ist ein deutliches Signal, dass Themen der Naturheilkunde und Komplementärmedizin so publiziert werden (können), dass sie spürbare «Aussenwirkung» entwickeln. Die Herausgeber investieren viel Zeit und Mühe, um im Dialog mit den Autoren jede Publikation inhaltlich und redaktionell zu optimieren, zum Vorteil der Autoren selbst (Impact-Faktor!) und der Sache (Impact!).

K.L. Resch, Bad Elster

\section{Literatur}

1 http://www.isinet.com/isi/hot/essays/journalcitationreports/7.html

2 Hansson S: Impact factor as a misleading tool in evaluation of medical journals. Lancet 1995;346: 906.
3 Winkmann G, Schlutius S, Schweim HG: Publikationssprachen der Impact-Faktor-Zeitschriften und medizinischen Literaturdatenbanken. Dtsch Med Wochenschr 2002;127:131-137.
4 Frömter E, Brähler E, Langenbeck U, Meenen NM, Usadel KH: Das AWMF-Modell zur Evaluierung publizierter Forschungsbeiträge in der Medizin. Dtsch Med Wochenschr 1999;124:910-915. 